\title{
La Didáctica de la Literatura en Argentina: de intervenciones fundacionales y mediaciones democráticas'
}

\author{
The teaching of literature in Argentina. Foundational \\ interventions and democratic mediations
}

\author{
ÁNGELES MARÍA INGARAMO \\ Universidad Nacional del Litoral \\ Argentina \\ angieingaramo@hotmail.com
}

Resumen. En el año 1989 se editó en Argentina La trama de los textos. Problemas de enseñanza de la literatura de Gustavo Bombini. El libro abordaba una serie de "obstáculos epistemológicos" (cf. Gerbaudo 2006, 20 I I; cf. Camilloni, 1997; Bachelard, 1948) presentes en la enseñanza escolar de la literatura, retomando distintas teorías que venían renovando los Estudios Literarios en el país. Al mismo tiempo, prefiguraba las líneas investigativas que habrían de consolidarse en el campo de la Didáctica de la Literatura a nivel local durante los años venideros.

En las recapitulaciones de esta Historia, surge este escrito, pretendiendo dar cuenta del lugar fundante e ineludible que ocupa el citado libro en los avances sobre la enseñanza de la literatura en Argentina.

Palabras clave: enseñanza de la literatura;teoría literaria; crítica literaria;Argentina.
Abstract. Bombini Gustavos's "The plot of texts. Problems of teaching literature" was published in Argentina in 1989. The book dealt with a series of "epistemological obstacles" (cf. Gerbaudo 2006, 20I I, cf. Camilloni, 1997; Bachelard, 1948) present in school teaching of literature, starting from the development of "mediation" (Gerbaudo, $20 \mathrm{I} \mathrm{Ia} \mathrm{,} \mathrm{20I} \mathrm{Ib),} \mathrm{based} \mathrm{on} \mathrm{a} \mathrm{number} \mathrm{of}$ theories that were to renew the field of literary studies.

At the same time, the text prefigured investigative avenues that should be consolidated in the area of the Teaching of Literature at a national level over the years to follow.

This article attempts to account for the foundational and essential place occupied by the said book in later developments of the field.

Key words: teaching literature; literary theory; literary criticism; Argentina.

\footnotetext{
I Para citar este artículo: Ingaramo, Ángeles (2OI2). La didáctica de la literatura en Argentina: de intervenciones fundacionales y mediaciones democráticas. Alabe 6. [www.revistaalabe.com]

(Recibido O2-O4-2OI2; aceptado I3-IO-2OI2)
} 


\section{Génesis y desarrollo de una esperada publicación}

El año I983 se presenta como un momento particularmente ineludible para quien pretenda un acercamiento a la historia argentina. Finalmente, luego de siete sangrientos años bajo el yugo del terrorismo de Estado, el país volvía a contar con un gobierno democrático.

La reinstalación de la mentada modalidad política no tardó en impactar en el estado del "campo intelectual" (Bourdieu, I992) del momento. Laxada oficialmente la soga de la censura, muchas voces anteriormente silenciadas o relegadas al ámbito de la clandestinidad comenzaron a florecer en el interior de este espacio.

El "campo literario" (Bourdieu, I992), por su parte, no se mantuvo ajeno a estas renovaciones. A este respecto, los estudios de Analía Gerbaudo (20ma) identifican en la coyuntura histórica postdictatorial la emergencia y consolidación de distintos "modos de leer” (Ludmer, r984; cf. Bombini, ı989; cf. Capdevila, ı997; cf. Biselli, r997) que habían quedado circunscritos durante "los años del terror" a lo que se denominó "universidad de las catacumbas": un espacio clandestino, paralelo al universitario oficial, donde distintos intelectuales importaban y traducían perspectivas teórico-literarias alternativas a las oficiales.

Con la venida de la democracia, varios de los sujetos integrantes de estos grupos de estudio, tales como Beatriz Sarlo, Josefina Ludmer, Enrique Pezzoni o Jorge Panesi, comenzaron a (re)ingresar al plantel docente de la universidad pública, introduciendo dichas perspectivas en el armado de aulas que contaban entre sus destinatarios con futuros profesores.

En este punto, para quienes abocamos nuestras investigaciones al ámbito de la didáctica de la literatura y que reconocemos en los estudios literarios uno de sus campos referenciales por excelencia (Gerbaudo, 2006, 20IIb; Bombini, I989, I992, I995, I996, 2005), surge la inevitable pregunta por los impactos de estas transformaciones específicas en el área de nuestro interés.

Contrariamente a lo que cabría esperarse de un período en el que se asistía a una dionisíaca renovación de saberes, no será sino hasta el año I989 cuando se publique en el país un texto que se proponga la democratización crítica de la citada renovación teórica para un público especializado en la enseñanza escolar de la literatura. Titulado La trama de los textos. Problemas de enseñanza de la literatura ${ }^{2}$ y escrito por Gustavo Bombini -por aquel entonces un joven docente de Escuela Secundaria, exalumno de las cátedras

\footnotetext{
${ }^{2}$ De aquí en más La trama de los textos...
} 
universitarias en las que se producían las citadas innovaciones, e incipiente Ayudante en la asignatura "Didáctica especial y prácticas de la enseñanza” de la carrera de Letras en la universidad de Buenos Aires-, el libro contó con un arrollador éxito de ventas.

Agotados los números de su primera edición que data de I989, el texto se volvería a publicar dos años después (I99I), ahora con una entusiasta tirada de 3 ooo ejemplares que doblaba en número a la primera. El dato no resulta menor si tenemos en cuenta que el ingreso de esta obra a la universidad, -uno de los centros por excelencia de multiplicación de lecturas (Dalmaroni, 2OII)- se fecha fundamentalmente desde el año I992 en adelante. ¿Quiénes eran los lectores que en sólo dos años habían agotado los I5oo ejemplares a la venta? ¿Quiénes eran aquellos para quienes, en un principio, se editaban las zooo copias más?

El citado desfase temporal que se establece entre la enunciación de la voz en i989 y su eco universitario recién en I992, habilita la reconstrucción del posible perfil consumidor del texto durante sus primeros años de circulación (I989-І99I). Se trataría del docente de literatura de nivel secundario en ejercicio, quien, al guiarse por el dato de la profusa reedición, se sentía si no representado, al menos interpelado por esta obra que lo tenía por su lector modelo.

La inmediata popularidad que adquirió el libro invita a ser comprendida en vinculación directa con dos hechos específicos. Por un lado, con el renombre que para aquel entonces venía adquiriendo el autor, a raíz de una serie de cursos de capacitación docente que desde el año i986 dictaba en distintos puntos del país, en compañía de María Adelia Díaz Rönner ${ }^{3}$. Por otro lado, con la situación desesperante de deslegitimación social y desprotección teórica en la que se hallaban los docentes escolares durante dicho período, una situación que los arrojaba hacia la propia búsqueda bibliográfica y auto-capacitación.

Para aquel entonces, el estado de los contenidos escolares era tan alarmante como la situación de la educación pública en general. Deserción, deterioro y salarios paupérrimos eran las caras visibles del saldo que había dejado el paso del gobierno dictatorial por el sector. Nada latente parecía resurgir con fuerzas de las iniciativas impulsadas durante los años 6 o y zo que se habían propuesto la renovación de las normas, contenidos y rituales escolares. Tampoco el Congreso Pedagógico Nacional celebrado por parte del Gobierno en el año i984 había aportado soluciones concretas para alcanzar la ansiada mejoría. Sumado a esto, muchos de los programas que se proponían la actualización y

\footnotetext{
3 Precisamente, el primero que ofrecen en forma conjunta en la ciudad de Mar del Plata llevó por título La trama de los textos, descubriendo así su calidad de antecedente inmediato para el texto en cuestión. Asimismo, no podemos negar la importancia que adquiere la citada autora en la génesis del texto. En la reedición de La trama de los textos... del año 2005 se lee en una parte de su Dedicatoria: Para María Adelia Díaz Rönner, quien resulta y confiada, me pidió en el año 1988 "setenta páginas sobre lo que vos hacés" y eso dio origen a mi primer libro. (Bombini, 2009: 3)
} 
capacitación del cuerpo docente habían quedado interrumpidos o sin concretarse en un país con su economía en pique. (Puiggrós, 2009: I8I)

En el estado anquilosado en el que se hallaba el sistema, la enseñanza escolar de la literatura había quedado subsumida en una visión enciclopedista de la historiografía lteraria (Bombini, r989) que reducía la tarea interpretativa de la lectura a la dilucidación de un único sentido dictaminado por la figura del autor, (Bombini, r989; Caisso y Rosa, I987). Al mismo tiempo, la cartelera de opciones bibliográficas quedaba circunscrita a un canon escolar determinado por las nociones de "literatura" como "modelo de buen decir" y "modelo de buen hacer", (Bombini, I989). Perdidos en tierra de nadie, los docentes de lengua y literatura de la escuela secundaria argentina parecían encontrar en los postulados de este libro una salida ante una práctica que estaba perdiendo toda su legitimidad social.

Con llamativa celeridad el éxito de La trama de los textos... comenzó a trascender los espacios privados, ingresando en los programas de los Institutos Terciarios de Formación Docente y, con mayor posterioridad, en los de la Universidad Pública Argentina4. En esta línea, se constata que desde el año I996 el texto se halla instalado en la totalidad de los programas de las cátedras de Didáctica Específica o afines, de la carrera de Letras de las principales universidades del país (UBA, UNC, UNLP, UNT), una incorporación que se perpetúa hasta la actualidad y que sigue provocando el "envío" (Gerbaudo, 2OIrb) a su adquisición y lectura.

Años luego, en 2005, se producirá una nueva reedición del texto que contará ahora con anotaciones por parte del autor al final de los apartados originales. Algo movía a Bombini a reescribir la trama, a resignificar sentidos, a reencontrarse con el que fue.

El libro, que "deviene otro como quien esto está escribiendo" (Bombini, 20o9: I25), ponía en diálogo dos proyecciones de un mismo sujeto que, si bien mostraban cierta continuidad, también manifestaban algunas divergencias. Entre un texto y el otro mediaban dieciséis años y uno de los sucesos más contraproducentes para la Educación

\footnotetext{
4 Valga a este respecto una anécdota ilustrativa de la buena acogida que recibió el texto en espacios destinados a la formación docente. Para el año I99o, el reconocido tallerista y profesor Nicolás Bratosevich, encontrándose a cargo de un Seminario sobre Teoría Literaria y Educación que se impartía en el marco de la "Maestría en Ciencias del Lenguaje" del Instituto Terciario "Joaquín V. González", organizaba su programa recuperando los aportes del texto para alcanzar una "actualización de criterios teórico-críticos en la consumición-producción literarias, aprovechable en el ámbito educativo", (Bratosevich, I99o). Toda una postura. Bratosevich no aspiraba a la mera reproducción mecánica de los conocimientos derivados de la Teoría y Crítica Literarias en el ámbito educativo, sino a su aprovechamiento, es decir, a la obtención de algún tipo de beneficio a partir de su uso.

La apuesta de La Trama de los textos... respondía a dicha exigencia, en tanto que recurría a los saberes teóricocríticos, no era para su simple transcripción en un libro destinado a docentes de Escuela Secundaria, sino para la elaboración de "mediaciones" (Gerbaudo, 2OII; 2OII) atentas a las problemáticas que estaban obstaculizando las prácticas.
} 
Argentina: la sanción de la Ley Federal N² 24I95. Con la aplicación de este régimen se clausuraba una etapa en la enseñanza de la literatura escolar caracterizada por el abordaje de los textos literarios como modelos del "buen decir" y del "buen hacer". En su lugar, las propuestas curriculares oficiales -y la mayoría de las editoriales- planteaban la consideración del objeto como "un discurso social más" (Gerbaudo, 20o6; Bombini, 2009), destinado a un tipo de placer asociado al facilismo, (Gerbaudo, 2006).

Bombini, portador de una visión compleja sobre el objeto, en tanto que "discurso estético de alta complejidad que requiere del desarrollo de múltiples competencias para ser abordado" (Bombini, I989: 25), desdeñará este tipo de proposiciones que en nombre del placer de la lectura borran todo tipo de esfuerzo interpretativo. A este respecto, el autor remarcará que la escuela "no puede dejar al sujeto lector sin herramientas válidas para comprender más y por ende para construir su propia experiencia de placer”, (Bombini, 2009: $3^{8) \text {. }}$

De cara a las ruinas que dejó una ley sagaz y egoísta, el autor intensificará en el año 2005 el reclamo de recuperar los saberes del sector docente para reinventar la enseñanza de la literatura, abandonando las recetas prescriptivas que les niegan su carácter de profesionales críticos.

Cuatro años luego, en 2009, La trama de los textos... se volvía a editar una vez más, lo que dejaba en claro que veinte años después de su primera aparición, el texto aún continuaba y aún continúa cautivando lectores.

\section{Una "mediación" fundante ${ }^{5}$}

La publicación de La Trama de los textos... invita a ser comprendida como un punto de inflexión en el curso de la historia de la Didáctica de la Literatura a nivel local. Con una originalidad inédita, el texto no sólo trazaba el delineado de una futura agenda investigativa atenta a los problemas y particularidades propios del sector; sino también, lograba detectar e intervenir en torno a ciertas problemáticas vigentes en la enseñanza escolar de la literatura a partir del diálogo con las nuevas, ignoradas o silenciadas voces provenientes de los estudios literarios.

En cuanto a lo primero, una mirada en retrospectiva permite corroborar el lugar fundante que adquiere la obra en el desarrollo de ciertas tendencias investigativas que posteriormente se afianzarán enérgicamente en el campo.

\footnotetext{
5 A partir del concepto de “mediación”, Gerbaudo (2OIIb) busca destacar la dimensión artesanal y subjetiva que posee la práctica de construcción de un discurso -el que acontece en la enseñanza de la literatura, o en el hacer propio de la Crítica Literaria- que lee, selecciona, tacha, reelabora los demás discursos, para elaborar el propio.
} 
Así, la preocupación manifestada por el autor por la figura del alumno pensado como lector será abordada en el país por un gran número de investigaciones en los años subsiguientes a la edición del texto. Tal es el caso de los estudios de Carolina Cuesta (200I, 2006), Liliana Peralta (200I), Elba Amado (2004), Mirta Fernández (2006) o Paula Labeur (2002), por sólo citar algunos casos.

Lo mismo ocurrirá en torno a la línea de investigación que funda el texto en una "zona de borde disciplinar"6 (Gerbaudo, 2006; 20mb) que se gesta entre la Teoría Literaria, y la Didáctica de la Literatura; una corriente atenta a la producción de "mediaciones" entre estos campos, en la que se destacan los trabajos de Analía Gerbaudo (2006; 2OIb), Miguel Dalmaroni (2006; 20II) y Daniel Link (I994), entre otros.

Finalmente, destacamos la importancia que Bombini le asigna en este texto a la narración de las propias prácticas por parte de los docentes, un modo de construcción de conocimiento que los reconoce como portadores de saberes derivados de la experiencia áulica con el objeto. Siguiendo los análisis de Gerbaudo (2009) sobre las perspectivas dominantes de trabajo que se vienen presentando en los principales congresos y encuentros del área a nivel nacional, este modo ha mostrado un copioso desarrollo en las últimas décadas.

Por su parte, en lo que respecta a la operación de "mediación" que realiza el texto en torno a los saberes que se derivan del campo de los estudios literarios, su abordaje obliga a remitirnos a los años de formación universitaria del autor.

Seguramente, de críticos y profesores claves en el momento en que cursaba sus estudios o trabajaba como docente en la Universidad de Buenos Aires como Beatriz Sarlo, Jorge Panesi, Enrique Pezzoni o Josefina Ludmer, Bombini había aprendido que la Teoría Literaria habría de servir como un dispositivo para leer mejor y no como conocimiento destinado al mero "aplicacionismo", (Bombini 1995; Gerbaudo 2006, 2OIIb). En dicha clave de lectura uno leía para decir otra cosa, para abrir paso a la novedad. En este caso, el autor se servía de los postulados teóricos que habían renovado el campo de los Estudios Literarios en Argentina precisamente para hacer otra cosa: detectar problemáticas y proponer posibles salidas para una práctica de enseñanza que había quedado obsoleta perdiendo su legitimidad social.

Gran conocedor del espacio educativo, afanoso lector de las renovaciones de la Teoría y Crítica literarias en el ámbito local y experto en los avances en el campo de la Di-

\footnotetext{
${ }^{6}$ Gerbaudo utiliza el concepto de "zona de borde" para referirse al entramado disciplinar que se vuelve necesario "para abordar problemas de la esfera educativa cuya complejidad impide que una sola pueda abarcarlos, demandando una confluencia teórico-epistemológica (...)" (2OIIb: 24)
} 
dáctica de la Literatura en el escenario internacional (Bombini, I992), Bombini se movía con soltura entre estos espacios del saber, haciendo un trabajo de traducción, probando alcances bibliográficos, detectando posibles cruces capaces de resolver las problemáticas que estaban obstaculizando las prácticas.

De esta forma, La Trama de los textos... fue inaugurando paulatinamente una "zona de borde disciplinar" que volvía a la renovación de la enseñanza escolar de la literatura una tarea de responsabilidades compartidas. Por un lado, la Didáctica de la Literatura debía abrirse a la reflexión teórico-literaria, -abandonando la idea vana de una práctica sin teoría (Bombini, r996)-; y por el otro, la Academia debía democratizar sus actualizaciones, elaborando "mediaciones” para un público más amplio que sí misma (Bombini, I995).

Teniendo en cuenta que la Escuela parecía haber quedado fuera del movimiento de traducción e importación de Teorías Literarias que venía ocurriendo en el "campo literario", la labor que emprendía el texto poseía un sesgo democratizador considerable: Bombini abría su biblioteca en búsqueda de posibles respuestas para una generación de docentes que, formada durante la Dictadura Militar y en ejercicio durante un gobierno poco dispuesto a prestar soluciones radicales para las problemáticas educativas, no había tenido posibilidad de actualizar profundamente sus conocimientos en el área. El texto arremeterá entonces en esta dirección, dirigiendo a sus lectores a profusos “envíos” bibliográficos que traducen un canon particular de lectura.

En este sentido, una presencia dominante en el mismo será la de la reconocida crítica literaria y profesora Josefina Ludmer. La trama de los textos... retomará varios de sus aportes, especialmente los derivados de un seminario sobre Teoría Literaria dictado por la autora entre los años I984 y I988, en el marco de la Facultad de Filosofía y Letras de la Universidad de Buenos Aires. El curso, concebido por Bombini como un punto de inflexión en el desarrollo del campo de la Teoría Literaria en la Argentina, se presenta aquí como el "seminario que habría que pensar como punto de viraje (...) en una cantidad de problemas que (...) tienen que repercutir o sería deseable que repercutieran en el interior de la práctica de la enseñanza" (Bombini, ı989: 43).

Recuperando sus reflexiones en torno a las funciones de la Teoría Literaria y haciéndose de la categoría de "modos de leer" postulada por la autora, Bombini se proponía lidiar con uno de los mayores “obstáculos epistemológicos” (Bachelard, I948; Camilloni, I997; cf. Gerbaudo, 20IIb) que afectaban a esta práctica: el de la concepción y presentación escolar de las conceptualizaciones literarias como universales y eternas. Para esto, decide contraponer la noción de literatura como "modelo del buen decir" a la performance de textos literarios que, si bien alcanzaban una clara presencia en el campo literario del momento, permanecían ajenos al canon escolar. En el ruido que generaba la falta de 
correspondencia entre el objeto y la conceptualización escolarmente adoptada, se volvía patente que los "modos de leer" respondían a la emergencia de producciones literarias específicas en tiempos y espacios determinados. De esta forma, el reconocimiento del carácter histórico que entrañan indefectiblemente las conceptualizaciones sobre lo literario, le permitía al autor explicar por qué ciertas aproximaciones nos permiten mirar mejor determinados textos, pero muestran sus claras limitaciones cuando las pautamos para el abordaje de otros. Fiel al objeto y sus alcances, Bombini se inclinará entonces por concepciones más bien "relacionales" (Vaccarini, 2008) sobre la literatura, "enviando" a sus lectores a textos como Estructuras y funciones del discurso de Teun Van Dijk o Escritos de estética y semiótica del arte Jan Mukarovsky, los que, si bien reconocen una especificidad estética en el objeto, la comprenden en determinación directa con lo que se concibe como tal en un momento social dado.

Otra presencia a destacarse será la de Beatriz Sarlo, una célebre analista de la cultura y crítica literaria que, al igual que Ludmer, ejercía la docencia universitaria durante el período de docencia y formación del autor en la universidad de Buenos Aires. La trama de los textos... no sólo "envía" explícitamente a ciertos libros que esta autora produjo en compañía de Carlos Altamirano como Literatura/sociedad (I977), o Conceptos de sociología literaria (I980); sino también a escritos que la Crítica Literaria identificará posteriormente como pertenecientes al "canon Sarlo", ya sea por la asiduidad con la que la autora los cita en sus trabajos, o por la responsabilidad directa que ejerció en su traducción e introducción en el escenario local. Tal resulta ser el caso de Manuel Puig y Juan José Saer, desde el ámbito literario; o a Mijaíl Bajtín, Pierre Bourdieu, Terry Eagleton, o Jan Mukarovsky, desde la Teoría y la Crítica Literarias.

A este respecto, interesa especialmente la recuperación que se realiza en el texto del concepto de "campo intelectual", una categoría postulada por Pierre Bourdieu (I992), importada al país fundamentalmente por la autora, la cual se retomaba aquí para proponer alternativas a los modos ingenuos y simplificadores de presentar la relación "texto-contexto" en el interior de las "aulas de literatura" escolares (Gerbaudo, 2OII). En este sentido, las referencias que se realizan al concepto van normalmente acompañadas por notas al pie que dirigen al lector a los escritos de Bourdieu, pero también a los de Sarlo, transparentando con este último gesto el valor que se le reconoce a esta reescritura teórica que imprime sobre el objeto sus propias marcas desde la perspectiva local.

En esta misma línea, también habría que mencionar la recurrencia en el texto a los postulados de Terry Eagleton -una presencia claramente asidua en los escritos de Sarlo de aquellos años-, cuyos aportes serán utilizados aquí para incitar la apertura escolar a la figura del "lector", tanto como dimensión textual que construye el texto, como cual sujeto real capaz de asignar sentidos en el proceso de lectura. 
De este modo, dando cuenta de la situación comunicativa concreta en la que siempre tienen lugar los “discursos" (Van Dijk, I996) literarios, Bombini buscaba subsanar otro de los grandes problemas que detectaba en la enseñanza escolar de la literatura: el de concebir al autor como garante del sentido último del texto. En los nuevos-tiemposdemocráticos que acontecían, se volvía necesario darle nacimiento al lector escolar, abrir los múltiples sentidos del texto, arriesgarse a modos más democráticos en el aula, entender al sujeto alumno como constructor de lecturas.

Algo quedaba en claro de todas estas apuestas teóricas, Bombini encontraba en los planteos del universo de la Teoría y Críticas Literarias modos ineludibles para la desarticulación de los mandatos escolares sobre la enseñanza de la literatura que obstaculizaban los alcances del objeto en la práctica de enseñanza. ${ }^{7}$

\section{Conclusión}

La publicación en el año I989 de La trama de los textos... de Gustavo Bombini supuso un punto de inflexión en la historia de la Didáctica de la Literatura en Argentina. El texto no sólo fundó y direccionalizó las principales líneas investigativas desarrolladas posteriormente en el campo, sino que también democratizó una serie de renovaciones teórico-críticas literarias que posibilitaron la revisión de ciertos supuestos imperantes en la enseñanza escolar de la literatura. En la tarea de abordar dichos supuestos críticamente, el texto diagnosticaba el estado de esta enseñanza, constituyéndose así como un testimonio en pie sobre nuestro pasado educativo-político. Una mirada en retrospectiva así parece corroborarlo.

\footnotetext{
${ }^{7}$ Esto se explica a partir de la visión que el autor poseía sobre la Teoría Literaria, a la que entendía como un cuerpo de saberes capaz de desbaratar las ideologías arraigadas, provocar la revisión del canon y habilitar la reflexión sobre los límites que se dibujaban entre los discursos considerados literarios y los que no. (De Man; cf. Bombini I996a) Desde esta lógica, la recurrencia a este campo de conocimientos se explica fáculmente, en tanto que las citadas operaciones se reconocían como tareas pendientes en lo que respecta a la enseñanza escolar de la literatura.
} 


\section{Bibliografía}

-Altamirano, C. (ig86). El intelectual en la represión y en la democracia. Punto de vista, 25, I-4.

- Altamirano, C. y Sarlo, B. (r977). Literatura/sociedad. Bs. As.: CEAL. ( (1980). Conceptos de sociología. Bs.: CEAL.

- Amado, E. (2004). Hacia una didáctica social: la formación del lector. En AA.VV. De las raíces hacia las alas. Tucumán en tiempos de lectura. Segundo Dossier. San Miguel de Tucumán: Secretaría de Estado de Educación.

- Bachelard, G. (I980). La formación del espíritu científico. México: SigloXXI.

- Biselli, R. (г997). Reflexiones en torno al discurso didáctico sobre la literatura: bibliografía comentada. En AA.VV. La enseñanza de la literatura como problema. (pp. 35-43). Rosario: UNR.

- Bombini, G. (1989). La trama de los textos. Problemas de la enseñanza de la literatura. Bs. As.: Libros del Quirquincho.

- (Coord.) (1992). Literatura y educación. Bs. As.: CEAL.

- --_(1995). Otras tramas. Sobre la enseñanza de la lengua y la literatura. Rosario: Homosapiens.

- - - (1996). Didáctica de la literatura y teoría: apuntes sobre la historia de una deuda. Orbis Tertius. Revista de teoría y crítica literaria, 2-3, 2II-2I7.

- -- (2009). La trama de los textos. Problemas de la enseñanza de la literatura.

Bs. As.: Lugar.

- Bourdieu, P. (1992). Las reglas del arte. Barcelona: Anagrama.

- Buchbinder, P. (2005). Historia de las universidades argentinas. Bs As.: Sudamericana.

- Caisso, C. y Rosa N. (1987) La enseñanza de la literatura en argentina. En AA.VV. (I997). La enseñanza de la literatura como problema. (pp. 7I-90). Rosario: UNR.

- Camilloni, A (Comp.) (i997). Los obstáculos epistemológicos en la enseñanza. Barcelona: Gedisa.

- Capdevila, A. (i997). La enseñanza de la literatura como problema teórico. En AA.VV. La enseñanza de la literatura como problema. (pp. 9-23). Rosario: UNR. 
- Cuesta, C. (200I). Hacia la construcción de una nueva mirada sobre los lectores y la literatura. Lulú Coquette. Revista de Didáctica de la Lengua y la Literatura, I, 9-3I.

- --_.-- (2006). Discutir sentidos: la lectura literaria en la escuela. Bs. As.: Libros del Zorzal.

- Dalmaroni, M. (2006). Una república de las letras. Lugones, Rojas, Payró. Escritores argentinos. Rosario: Beatriz Viterbo.

- - - (2OII). La crítica literaria y el sujeto secundario. Panfleto sobre un modo de intervención subalterno. El toldo de Astier. Propuestas y estudios sobre la enseñanza de la Lengua y la Literatura, 2, I-II. Obtenido el o8 de Marzo de 2012 desde http://www.eltoldodeastier.fahce.unlp.edu.ar/numeros/numero-2/m-dalmaroni-nro-2.pdf/view.

Fernández, M. (2006). ¿Dónde está el niño que yo fui? Adolescencia, literatura e inclusión social. Bs. As.: Editorial Biblios.

- Gerbaudo, A. (2006). Ni dioses ni bichos. Profesores de literatura, currículum y mercado, Santa Fe: UNL.

- - (2009). Literatura y enseñanza. En Dalmaroni, M. (Dir.) La investigación literaria. Problemas iniciales de una práctica (pp. I47-I82). Santa Fe: UNL.

- - (20IIa). La literatura en la universidad argentina (I984-I986). Intervenciones desde una política de la exhumación. Moderna Sprak, 2. Obtenido el o8 de Marzo de 2OI2 desde http://ojs.ub.gu.se/ojs/index.php/modernasprak/article/view/948

UNL, Homo Sapiens.

- Giménez, G. (1997). Literatura y enseñanza: una relectura política de la teoría. En Bombini G. (Coord) (1997). Voces de un campo problemático. Actas del I Congreso Nacional de Didáctica de la Lengua y la Literatura (pp. I27-I36). La Plata: UNLP.

- Labeur, P. (2002). Otra carta robada: prácticas de escritura de textos académicos en el nivel superior. En Boletín de Humanidades. Bs. As.: Nueva época.

- Link, D. (I994). La chancha con cadenas. Bs. As.: Ediciones del Eclipse.

- Mukarovsky, J. (200o). Escritos de estética y semiótica del arte. Santa Fe de Bogotá (Colombia): Plaza y Janés.

- Peralta, L. (200I). Escuela y escritura. Una dupla problemática. Lulu Coquette. Revista de Didáctica de la Lengua y la Literatura, I, II2-I7. 
- Puiggrós, A. (2009). Quépasó en la educación argentina. Breve historia desde la conquista hasta el presente. Bs. As: Galerna.

- Vaccarini, C. (2008). Literatura. En Amícola, J. y de Diego, J.L. (Coords.) La teoría literaria hoy. Conceptos, enfoques, debates (pp. II-22). Bs. As.: Al margen.

-Van Dijk, Teun (1996). Estructuras y funciones del discurso. Mexico: Siglo XXI. 